\title{
A Computational Framework for Human/Agent Communication Using Argumentation, Implicit Information, and Social Influence
}

\author{
Jamal Bentahar \\ Concordia University, \\ Canada \\ bentahar@ciise.concordia.ca
}

\author{
Karim Bouzoubaa \\ Mohammadia College \\ Morocco \\ karim.bouzoubaa@emi.ac.ma
}

\author{
Bernard Moulin \\ Laval University \\ Canada \\ bernard.moulin@ift.ulaval.ca
}

\begin{abstract}
In this paper, we propose a new computational framework for human/agent communication. The main objective is to allow software agents to participate in flexible communications with human and to be efficient in these communications. To be flexible and efficient, artificial agents in our model are able to: 1) deal with the implicit aspects of conversations by considering the non literal level of speech acts; 2) reason on their internal states and on the conversation state using argumentation abilities; and 3) manage the social influence. Our framework is based on the philosophical foundations provided by speech act theory, argumentation theory, and social commitments.
\end{abstract}

\section{Introduction}

Recent years have seen an increasing interest in agent communication within multi-agent community $[1,8,9,11,15]$. Extending this kind of communication to human/agent communication is a challenging research problem. As outlined in [3], agents in such a communication should have some capabilities to understand and interpret natural languages. For this reason, speech act theory [6] can be used as a foundation since humans communicate by exchanging speech acts, which can be computed and then used by software agents. However, when analyzing human conversations, many practitioners of conversational analysis consider that speech act theory cannot be used in its current version and must be extended. Speech act theory is essentially a theory of analyzing isolated speech acts at their literal level without considering the social context and the participants' reasoning capabilities. To develop advanced and powerful models for flexible communications between humans and artificial agents, the sequencing of speech acts, their non-literal interpretation, and the social context in which they are performed should be addressed.
Some directions have been proposed to enrich the traditional version of speech act theory. Dascal [12] believes that it is its restriction to literalness that prevents the extension to conversation of traditional speech act theory. For him, pragmatic dimension is indispensable to account for the sequencing of illocutionary acts. For Van Rees [13], it is indispensable to take into account both communicative and interactive effects of every utterance in order to explain the achievement of a speech act in conversation. In a more detailed analysis of this aspect, Brassac [2] proposed a model that involves two extensions of speech act theory by introducing a dialogical perspective that transcends the monological limitations of the actual speech act theory and by considering essential the non-literal aspect of utterances. Vanderveken [6] proposed a formal theory to deal with the non literal meaning of utterances. For him, indirect speech acts are cases of exploitation of the maxim of quantity. In addition, for Vanderveken [5], speakers can make a linguistic exchange in order to fix together the interpretation to give to a previous utterance that is ambiguous or could be non literal. This leads Vandervken to enrich illocutionary logic so as to contribute to the foundations of the logic of discourse.

Thus, some fundamental points to consider when developing humanlike communication models are: the non literal level of speech acts (or indirect speech acts), the conversational sequencing, and the pragmatic level. To illustrate the problem, let us consider the following dialogue between a human user and an artificial agent.

(1) User: Agent!

(2) Agent: Yes, sir

(3) User: Can you send an email to Paul to let him know that I won't come for lunch and can you please also search the best price on internet for a Pentium IV

(4) Agent: How do you want me to send Paul the email?

(5) User: Be polite please, it's necessary to 
contact Adam also

(6) Agent: What should I tell him?

(7) User: No, no, I will contact Adam myself

It is easily observed from this simple dialogue that an agent involved in a conversation should reason on:

1. The indirectness level of speech acts: can you send an email to Paul?;

2. The understanding of the communicative intention: the utterance "it's necessary to contact Adam" is interpreted first by the agent as a directive until the user corrects this situation later in the conversation by telling "no, no, I will contact Adam myself";

3. The pragmatic level, especially the social one: the way the agent will contact Paul depends on the social relationship between the user and Paul.

In addition, in order to enable agents to communicate flexibly and efficiently with humans, they should be able to reason on their internal states (beliefs, intentions, goals, etc.) and on the communicative acts performed during the conversation. Communicating flexibly means that agents do not simply execute predefined (rigid) protocols, but decide about the response to give at runtime depending on the current state of the conversation. Communicating efficiently means that agents can use their reasoning capabilities to select an appropriate communicative act among other possible communicative acts. The model we propose in this paper addresses these fundamental aspects necessary to develop advanced and powerful agent/human communication. The objective is the automation of conversations between human and software agents by addressing four conversational phenomena: interpreting indirect speech acts, understanding communicative intentions, deciding about next communicative acts to be performed, and considering social influences. Semantically and technically speaking, our approach is different from the approaches used in collaborative dialogues proposed in $[4,7,10,14]$. The main differences are that unlike collaborative dialogue approaches, our approach uses an advanced reasoning allowing agents to argue about their choices, and a public social component reflected by social commitments and social relationships.

The reset of this paper is organized as follows. Section 2 presents the agent architecture we use in our framework. Section 3 stresses the foundations of the underlying communication model (social commitments and arguments). Section 4 addresses the solution we propose for implicit information. Section 5 explains how social influences are captured in our framework. Section 6 concludes the paper and identifies future work.

\section{Agent Architecture}

The artificial agent architecture we use in our framework is composed of three models: the mental model, the social model and the reasoning model. The mental model includes beliefs, desires, goals, etc. The social model captures social concepts such as conventions, roles, etc. Social commitments (SCs) made by agents when conversing are a significant component of this model. The agent's SCs reflect his private mental states. Thus, agents must use their reasoning capabilities to reason about their mental states before creating SCs. The agent's reasoning capabilities are represented by the reasoning model using an argumentation system. Argumentation is used by agents to reach a decision, to inform, and convince the human. Our conversational agent architecture also involves general knowledge, such as knowledge about the conversation subject. This architecture is motivated by the fact that conversation is a cognitive and social activity, which requires a mechanism making it possible to reason about mental states, about the current state of conversation (public aspects), and about the social aspects (conventions, obligations, etc).

In our software agent model, agents can also reason about their preferences in relation to beliefs. The idea is to capture the fact that some facts are more strongly believed (or desired). This mechanism allows agents to select an appropriate communicative act when several acts are possible. For this reason, we assume that any set of facts has a preference order over it. We suppose that this ordering derives from the fact that the agent's knowledge base denoted by $\Gamma$ is stratified into nonoverlapping sets $\Gamma_{1}, \ldots, \Gamma_{\mathrm{n}}$ such that facts in $\Gamma_{\mathrm{i}}$ are all equally preferred and are more preferred than those in $\Gamma_{\mathrm{j}}$ where $i<j$. We can also define the preference level of a subset of $\Gamma_{i}$ whose elements belong to different non-overlapping sets as follows.

Definition 1 (Preference level of a subset of knowledge base). The preference level of a nonempty subset $\gamma$ of $\Gamma$ denoted by level $(\gamma)$ is the number of the highest numbered layer which has a member in $\gamma$.

Example 1. Let $\Gamma=\Gamma_{1} \cup \Gamma_{2}$ with $\Gamma_{1}=\{a, b\}, \Gamma_{2}=\{c, d\}$, $\gamma \neq\{a\}$, and $\gamma=\{a, d\}$. We have level $(\gamma)=1$, level $\left(\gamma^{\prime}\right)=2$.

\section{Communicational Model}

At the semantic level, our communication model is based on the notion of SCs. A SC is a public engagement made by a human or artificial agent, that some fact is true or to do something [8]. SC can be viewed as a generalization of obligations as studied in 
deontic logic. Considering their deontic nature, SCs define constraints on the agents' behavior. SCs are social in the sense that they are publicly expressed, observed by all the participants, and governed by some rules. The main idea is that a speaker is committed to a statement when he made this statement or when he agreed upon this statement made by another participant, and acts accordingly. For example, by committing that a certain fact is true, the agent is compelled not to contradict himself during the conversation. He must also use his argumentation system to be able to explain, argue, justify and defend his commitment.

In order to model the dynamics of conversations, we interpret a speech act SA as an action performed on a commitment or on its content [9]. A speech act is an abstract act that an agent, the speaker, performs when producing an utterance $\mathrm{U}$ and addressing it to another agent, the addressee. The actions that an agent can perform on a commitment are: Act $\in$ \{Create, Withdraw $\}$. The actions that an agent can perform on a commitment content are: Act-cont $\in$ \{Accept, Refuse, Challenge, Defend, Attack, Justify $\}$. In our framework, a speech act is interpreted either as an action applied to a commitment, or as an action applied to its content. Formally, a speech act can be defined as follows.

\section{Definition 2 (Speech act). $S A\left(A g_{1}, A g_{2}, t, U\right)=_{d e f}$ $\operatorname{Act}\left(A g_{1}, t, S C\left(A g_{1}, A g_{2}, \varphi\right)\right)$ |Act-cont $\left(A g_{1}, t, S C\left(A g_{i}, A g_{j}, \varphi\right)\right)$ $A \operatorname{Act}\left(A g_{1}, t, S C\left(A g_{1}, A g_{2}, \varphi\right)\right) \&$ $\operatorname{Act}-\operatorname{cont}\left(A g_{1}, t, S C\left(A g_{i}, A g_{j}, \varphi\right)\right)$}

where $t$ is the utterance time and $\varphi$ is the commitment content. The definiendum $S A\left(A g_{1}, A g_{2}, t, U\right)$ is defined by the definiens $\operatorname{Act}\left(A g_{1}, t, S C\left(A g_{1}, A g_{2}, \varphi\right)\right)$ as an action performed by the speaker on its SC. The definiendum is defined by the definiens $\operatorname{Act}-\operatorname{cont}\left(A g_{1}\right.$, $\left.t, S C\left(A g_{i}, A g_{j}, \varphi\right)\right)$ as an action performed by the speaker on the content of its $\mathrm{SC}(i=1 ; j=2)$ or on the content of the addressee's SC $(i=2 ; j=1)$. Finally, the definiendum is defined as an action performed by the speaker on its $\mathrm{SC}$ and as an action performed by the speaker on the content of its $\mathrm{SC}$ or on the content of the addressee's SC.

In order to support their actions performed on commitments and commitment contents, agents use their argumentation systems. An argumentation system essentially includes a logical language $L$, a definition of the argument concept, and a definition of the attack relation between arguments. Here $\Gamma$ indicates a knowledge base with deductive closure. $\therefore$ stands for classical inference.
Definition 3 (argument). An argument is a pair $(H, h)$ where $h$ is a formula of $L$ and $H$ a subset of $\Gamma$ such that: i) $H$ is consistent, ii) $H \therefore$ h and iii) $H$ is minimal, so that no subset of $H$ satisfying both $i)$ and ii) exists. $H$ is called the support of the argument and $h$ its conclusion.

Definition 4 (attack). Let $(H, h),\left(H^{\prime}, h^{\prime}\right)$ be two arguments. $(H, h)$ attacks $\left(H^{\prime}, h^{\prime}\right)$ iff $H^{\prime} \therefore \neg h$.

In fact, before committing to some fact being true (i.e. before creating a commitment whose content is $h$ ), the speaker agent must use its argumentation system to build an argument $(H, h)$. The addressee agent must also use its own argumentation system to select the answer it will give (i.e. to decide about the appropriate manipulation of the content of an existing commitment). For example, an agent $A g_{1}$ accepts the commitment content $h$ proposed by another agent if $A g_{1}$ has an argument for $h$. If $A g_{1}$ has an argument neither for $h$, nor for $\neg h$, then it challenges $h$. Finally, we notice that when an agent $A g_{1}$ communicates its argument to the addressee, this argument becomes an $A g_{1}{ }^{\prime}$ commitment. Thus, the addressee can act on this argument as a commitment content.

Our human/agent communication model is based on the following fundamental principles:

- Communication is considered as a negotiation process of SCs. This process is formed by a set of initiative/reactive dialogue games.

- Communication results in a manipulation of SCs supported by arguments [8].

- Agents reason on their mental states and on the current conversation state to perform the next communicative act using an argumentative reasoning.

\section{Implicit Information}

It is easily observed that humans use indirect speech acts more frequently than direct speech acts. For instance, when a manager says to his secretary "Can you print the document number 5?" his utterance should be interpreted as a polite way of ordering her to print the document (non literal interpretation) and not as a question about her ability to print (literal interpretation). Also, the question asked by the user to his agent (in the first dialogue) "Can you send an email to Paul?" should be interpreted too as a directive speech act.

In order to take into account this conversational phenomenon, we suggested to model implicit information conveyed by speech acts. Given a speech act $S A$ performed by speaker $L_{1}$ and directed to interlocutor $L_{2}$, we define the implicit information 
conveyed by $S A$ as the information that $L_{l}$ intends to transfer to $L_{2}$ and which is different from $S A$ 's propositional content. For example, the implicit information associated with the question "Can you send an email to Paul?" is the request to send the email.

In order to provide a mapping between implicit and explicit information, we use knowledge structures called conversational schemas to interpret indirect speech acts. Conversational schemas specify conversational conventions that apply in a given socioorganizational context. A conversational schema can be used by an agent either for choosing a speech act that reflects its communicative intention, or for interpreting other agents' speech acts.

A conversational schema has the following form: CONV-SCH "ident";

Context;

Characteristics;

Communicative intention;

Explicit information;

Communicative Expectation

Each agent possesses a set of conversational schemas. This set represents its knowledge of the conversational practices of the society to which it belongs. A conversational schema is used depending on a certain Context. In our approach, this context is either a social or a personality context. The Characteristics slot has two components. The first one concerns the illocutionary strength, which is quantitative, and allows the agent to have different formulations for the same communicative intention. The second component is the refusal option that indicates if the agent can refuse a given directive. When an agent wants to express a certain Communicative intention, it chooses a conversational schema depending on the social and personality context. This conversational schema gives it the corresponding formulation in the Explicit information slot. The slot Communicative Expectation will be explained in the next section.

For example, the corresponding conversational schema for a "polite request" is formulated as follows: $\mathrm{CONV}$-SCH "polite request";

Context: social = peer, personality = any;

Characteristics: illoc-strength(0), refusal-option(yes);

Communicative intention: $\operatorname{DIR}\left(A g_{1}, A g_{2}, t\right.$, Prop $)$;

Explicit information: INQUIRE $\left(A g_{1}, A g_{2}\right.$, peer, $t$,

$H A S-C A P A C I T Y\left(A G T\left(A g_{2}\right), O B J(P r o p)\right)$, Yes/No?)

The above "polite request" conversational schema used by an agent $A g_{1}$ toward an agent $A g_{2}$, specifies that the social context should be a peer social power between the two agents and that agent $A g_{1}$ might have any kind of personality. This conversational schema has illocutionary strength of 0 and it concerns a directive speech act $(D I R)$, which gives a refusal option to the interlocutor. In this scheme, agent $A g_{1}$ has the intention to propose a directive to agent $A g_{2}$ and for this purpose, it will publicly perform an inquire (INQUIRE) asking agent $\mathrm{Ag}_{2}$ (AGT means agent) about its capacity to do the needed action expressed by Prop (OBJ means object). Indeed, explicit information indicates the action applied by the agent on a social commitment.

\section{Social Influence}

Social relationships influence the way people interpret indirect speech acts. It is easily observed that locutors are aware of the roles they play and the social power they have in the organizational setting in which a conversation takes place. A soldier cannot give orders to his general without violating the power scale established in the army. We can also notice that most often social relationships are not expressed in locutors' speech acts: they remain implicit. In certain cases however, when a social relationship is violated, locutors react and may decide to invoke their social relationships: hence they become explicit. Suppose that for whatever reason a secretary refuses to perform a task requested by her manager, the manager may strongly react saying: "I am your boss and you have to do what I order you to do".

In order to model the social context, in which agents evolve, we provide them first with specific mental states related to their inter-personal relationships: their roles and the social power induced by these relationships. A reasoning mechanism using these social mental states guides agents when selecting a conversational schema and allows them to perform a speech act with respect to the power relationship established with others.

In societies, human agents know the interpersonal relationships they may have with others. For example, a father knows that he has a "father-son" relationship with his son. However, this interpersonal relationship is not the same for every father-son couple but depends on the power that each agent has with respect to the other. For example, a father may have power over his son while another father may have a peer power relation with his son.

In addition, a human agent is aware of the role he plays with respect to a given interpersonal relationship. For this specific role, the agent is also aware of the conversational practices of his organization. In other words, an agent playing a certain role inside a given interpersonal relationship is aware of the conversational schemas that he can use. Hence, the following structures are used to specify interpersonal relationships and roles:

INTER-REL(Ident, Name, List of [role, power $])$ 
ROLE(Ident, Name, List of Conv. Schemas)

For example, the mental states structures associated with a father-son relationship might be:

INTER-REL(I1, father-son, [

(father-normal, son-normal, power(father $>$ son)),

(father-weak, son-strong, power(son>father $))]$ )

ROLE(I2, father-normal, [List2])

ROLE(I3, son-normal, [List3])

$\operatorname{ROLE}(I 4$, father-weak, [List 4$])$

ROLE(I5, son-strong, [List5])

In some organizations, the father-son relationship may be of two types. In the first type, the father plays a "father-normal" role and the son plays a "son-normal" role. In this couple, the father has power over his son. The father-normal is defined in the $I 2$ role structure, List 2 representing the list of conversational schemas that the father can use.

We need now a mental state, which specifies for a certain agent which social relationships he has with the other agents that it knows. For that purpose, we use the following structure:

SOCIAL-POSITION(Name of Inter-Rel,

(Agent 1 , role 1$),($ Agent 2 ,role 2$)$ )

Let us suppose that Adam is the father of Paul and that they have a normal father-son relationship. Hence, Adam has the following social position toward his son:

SOCIAL-POSITION(father-son,

(Adam, father-normal), (Paul, son-normal))

For Paul, his social position toward his father is:

SOCIAL-POSITION(father-son,

(Paul, son-normal), (Adam, father-normal))

When an agent does not respect the 'expected' social relationship, the other agent usually reacts to this anomaly either by acting in order to force the interlocutor to repair his misconduct, or by judging the sociability of the unrespectful agent. In order to elucidate the mechanism that allows an agent to reason about this phenomenon, let us consider and analyze the following dialogue:

(1) Father: Pass me the salt please.

(2) Son: No!

(3) Father: What?! I am your father and I order you to do it!

We suppose that the father and the son have the social positions described earlier. The dialogue can be detailed according to the father's point of view as follows:

We consider that the father is agent $A 1$ and that the son is $A 2$. At time $t 1$, the father is proposing a directive to his son using a "Request in a power position" conversational schema and he is expecting his son to accept:

- PROPOSE(A1, A2, $\operatorname{pow}(A 1>A 2), t 1$,
$\operatorname{DIR}(A 1, A 2, t 1, \operatorname{pass}(A G T(A 2, A 1), O B J($ salt $))))$

- $A C C E P T(A 2, A 1, \operatorname{pow}(A 1>A 2), t 2$,

$\operatorname{DIR}(A 1, A 2, t 1, \operatorname{pass}(A G T(A 2, A 1), O B J($ salt $))))$

At time $t 2$, the son is refusing the directive of his father. But, according to his social position relative to his father, this refusal means to the father (using a conversational schema: 'refusal of a request in unpower position') that his son is also proposing an assertive. The content of this assertive is a new social position in which the son will get more power inside their interpersonal relationship:

- $\operatorname{REFUSE}(A 2, A 1, \operatorname{pow}(A 1>A 2), t 2$, $\operatorname{DIR}(A 1, A 2, t 1, \operatorname{pass}(A G T(A 2, A 1), O B J($ salt $))))$

- PROPOSE(A2, A1, pow(A1>A2), t2, ASS(A2, A1, t2, SOCIAL-POSITION(father-son,

(Paul, son-strong), (Adam, father-weak))))

According to the definition of the father-son interpersonal relationship and according to the new social position proposed by the son, the social power between the father and the son would change to pow(son>father). The father instantly reacts to the his son's refusal and implicitly refuses the new social mental state proposed by his son. To show this, at time t3, he explicitly reminds his son their 'normal' social position and he uses a less polite conversational schema to express his first intention using a conversational schema 'refusal of a social challenge':

- $\operatorname{REFUSE}(A 1, A 2, \operatorname{pow}(A 1>A 2), t 3, A S S(A 2, A 1, t 2$, SOCIAL-POSITION(father-son, (Paul, son-strong), (Adam, father-weak)) ))

- $\operatorname{PROPOSE}(A 1, A 2, \operatorname{pow}(A 1>A 2), t 3$, $\operatorname{DIR}(A 1, A 2, t 3, \operatorname{pass}(A G T(A 2, A 1), O B J($ salt $))))$

\section{Conclusion and Future Work}

In this paper, we proposed a computational framework for human/agent conversation by extending and enriching some techniques used in multi-agent communication. In this framework, human/agent communication is abstracted as a negotiation process of social commitments. To be able to flexibly select the communicative acts to be performed, software agents can reason on their mental states and social commitments using their argumentation systems. In addition, agents can handle implicit information and social influence using conversational schemas. The implicit aspect is captured by taking into account the non literal level of speech acts.

The framework is implemented within a prototype called POSTAGE managing administrative correspondence by tacking into account the formulation rules used in a particular organization. Using POSTAGE, a user can formulate a message in an informal way. The POSTAGE agent will transform 
this message in a way which agrees with (1) the social relationship existing between the user and his addressee; (2) the user communicative intention and (3) the formulation rules used in a particular organization. For example, the informal message "You are laid off" would be transformed into "As general manager, I deeply regret having to announce your dismissal from our company". A POSTAGE agent has a specific architecture that allows it to perform the correspondence task. This architecture is divided into two parts. The first part includes four knowledge models and the second one three execution modules. The user's model contains knowledge concerning the user such as his/her preferences and his/her social relationships with other users. The static knowledge contains plans and specific formulation schemas. A formulation schema is used by the agent to find a natural language expression for a given conversational schema. The planning module allows the agent to create messages on the basis of the elements selected by the user. The task of the learning module is to learn new knowledge such as user's preferences or formulations used in a given organization. The prototype is implemented in Java and Jack Intelligent Agents and the underlying argumentation reasoning is implemented in a logical language. Figure 1 illustrates a snapshot of this prototype.

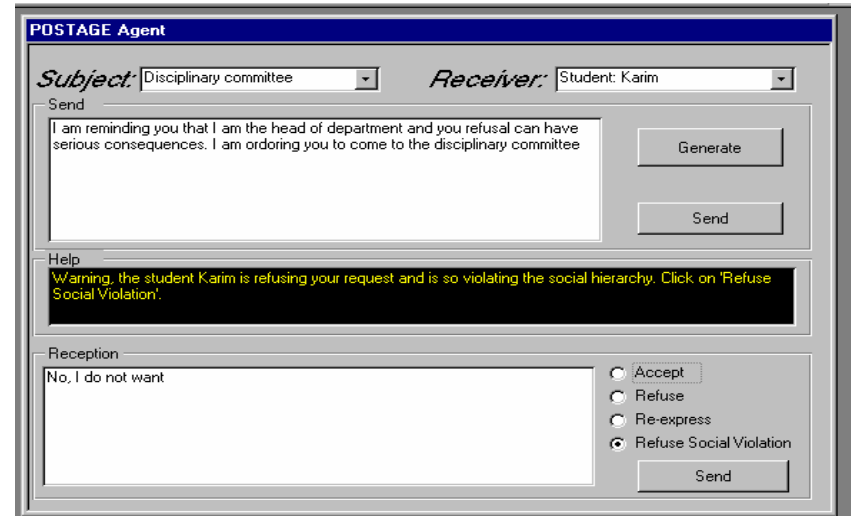

Figure 1. A POSTAGE snapshot

As future work, we intend to improve the framework by considering further social, cognitive, and linguistic foundations. We also plan to improve our prototype by using real corpora. Another issue for future work is to develop a general theory of dialogue games used to abstract the communication. This theory will allow agents to persuade each other, to negotiate with each other, etc. We also plan to define a formal semantics of the dialogue games. This semantics will be useful to develop formal verification of these dialogue games.

\section{References}

[1] B. Chaib-draa, and F. Dignum, "Trends in Agent Communication Language", Computational Intelligence, 18(2), 2002, pp. 1-14.

[2] C. Brassac, "Speech Acts and Conversational Sequencing", Pragmatics and Cognition, 2(1), 1994, pp. 191205.

[3] C. Kemke, "Natural Language Communication Between Human and Artificial Agents", Agent Computing and MultiAgent Systems, Springer, 4088, 2006, pp. 84-93.

[4] C. Rich, C. Sidner, and N. Lesh, "Collagen: Applying Collaborative Discourse Theory to HumanComputer Interaction”, AI Magazine, 22(4), 2001.

[5] D. Vanderveken, "Illocutionary Logic and Discourse Typology", Special Issue 216 Searle with his Replies of Revue Internationale de Philosophie, 2001, pp. 243-255.

[6] D. Vanderveken, "Meaning and Speech Acts", Principles of Language Use, Cambridge University Press, 1990.

[7] J. Allen, G. Ferguson, and A. Stent, "An Architecture for More Realistic Conversational Systems", Proc. of Intelligent User Interfaces, 2001, pp. 1-8.

[8] J. Bentahar, "A Unified Framework for the Pragmatics and Semantics of Agent Communication", Ph.D. Thesis, Laval University, Canada, 2005.

[9] J. Bentahar, B. Moulin, J-J. Ch. Meyer, and B. Chaibdraa, "A Logical Model for Commitment and Argument Network for Agent Communication", Proc. Of the 3rd Int. J. Conf. On AAMAS, IEEE Press, 2004, pp. 792-799.

[10] J. Rickel, N. Lesh, C. Rich, C. Sidner, and A. Gertner, "Using a Model of Collaborative Dialogue to Teach Procedural Tasks", Proc. International Conference on Artificial Intelligence in Education, 2001.

[11] K. Bouzoubaa, J. Bentahar, and B. Moulin, "Dialogization and Implicit Information in an Agent Communicational Model", Agent Communication, Springer, 3396, 2005, pp. $193-208$.

[12] M. Dascal, "On the Pragmatic Structure of Conversation", Searle on Conversation, compiled by $\mathrm{H}$. Parret and J. Verschueren, Amsterdam: John Benjamins, 1992, pp. 35-56.

[13] M.A. Van Rees, "The Adequacy of Speech Act Theory for Explaining Conversational Phenomena: a Response to Some Conversation Analytical Critics", Journal of Pragmatics, 17, 1992, pp. 31-47.

[14] O. Charles, and B. Grosz. "Interpreting Information Requests in Context: A Collaborative Web Interface for Distant Learning". Autonomous Agents and Multi-Agent Systems, 5, 429-465, 2002.

[15] R.M. van Eijk, M.-P. Huget, and F. Dignum, Agent Communication, Springer, 3396, 2004. 